\title{
As a Potentially Life-Threatening Disease; Cerebral Venous Sinus Thrombosis
}

\author{
Abdurrahman Sönmezler* \\ Assistant Professor, Department of Neurology, Adana City Research and Training Hospital, Adana,Turkey \\ *Corresponding Author: Abdurrahman Sönmezler, Assistant Professor, Department of Neurology, Adana City Research and Training \\ Hospital, Adana, Turkey.
}

Received: August 26, 2019; Published: September 13, 2019

DOI: 10.31080/ASNE.2019.02.0107

Cerebral venous sinus thrombosis(CVST) includes thrombosis of dural sinuses and/or veins. It constitutes less than $1 \%$ of all stroke and is usually seen in young people. More prevalence in young women is due to pregnancy, puerperium and oral contraceptive pills (OCP) use. CVST leads to infarction of the draining zone brain parenchyma. Patients usually present with headache, seizures, papilledema, altered consciousness, and focal neurological deficits. Headache is the most common patient presentation. The quality of this headache is highly variable with no specific location or pattern. The use of magnetic resonance imaging is more sensitive for diagnosis in all stages of thrombosis than the use of computed tomography. Digital subtraction angiography (DSA) is the traditional gold standard for the diagnosis of CVST, but nowadays MRI/magnetic resonance venography (MRV) is recommended by the European Federation of Neurologic Societies guidelines as the preferred brain image, whereas CT/CT venography (CTV) is an acceptable option when MRI is not available. Nevertheless, neither DSA nor MRV/CTV can provide the hemodynamic status of the cerebral veins and sinuses. Transcranial ultrasound provides information on venous hemodynamics, which is not provided by other neuroimaging modalities and is therefore complementary to these methods. Anticoagulants are the first preferred group of drugs. CVST is a very difficult disease to diagnose due to the variability in both patient presentation and imaging findings [1-4].

Cerebral venous system is divided into superficial and deep system. The deep system is composed of the lateral sinus, straight sinus, and sigmoid sinus along with draining deeper cortical veins. The deep medullary veins are located adjacent to the atrium and posterior body of the lateral ventricle. The deep medullary veins drain venous blood from the white matter towards the subependymal veins of the lateral ventricles. Under physiological conditions, these are small calibrated vessels that are difficult to demonstrate by conventional magnetic resonance imaging (MRI) techniques. Paramagnetic sensitive MR sequences (T2-weighted imaging) and susceptibility -weighted imaging (SWI) allow for better visualization of these vessels, especially when there is an increase in intravenous deoxshemoglobin. The brush sign (BS) is an abnormal hypointensity of the subependymal and deep medullary veins in paramagnetic-sensitive MR sequences defined in patients with ischemic stroke. The relationship between BS and the extent of thrombosis, parenchymal brain lesion, and clinical manifestations with focal neurological deficits suggests that BS may be a marker of severity in determining the increased intracranial venous pressure associated with CVST [5].

Extent of CVST does not determine clinical severity, MRI lesion, and outcome. This specified condition was identified in a recent study involving 160 patients. In this study, the extent of venous sinus thrombosis was scored based on the distribution of clots in the sinuses [2].

There are a wide range of causes in CVST etiology. Collagen tissue diseases, inflammatory diseases, genetic factors such as Factor V Leiden mutation, protein C-protein S-antithrombin 3 deficiency, prothrombin gene mutations, homocysteine elevation, cancerinduced hypercoagulability, antiphospholipid and anti-cardiolipin antibodies can be caused. Data in Asian countries compared with western countries important differences apeear as compared to western data including younger age, high frequency of anemia, low use of OCP, high frequency of hypercoaguable states, and a longer diagnosis delay. In spite of low number of diagnostic and treatment resources in Asian countries, overall outcome was good. Identification of high-risk patients, early diagnosis and aggressive treatment may improve outcome. In particular, a young female patient with headache, papilledema, seizures or focal neurological deficit 
should refer any physician to CVST. Public and physician awareness can play a key role in early diagnosis and treatment of these patients [6].

The results of a study investigating the relationship between clotting age and recanalization using Magnetic resonance blackblood thrombus imaging (MRBTI) in patients with severe CVST treated with endovascular treatment were promising. MRBTI has recently been proposed as a noninvasive imaging tool for direct thrombus imaging. Acute thrombi consisting of deoxyhemoglobin presented as an isointense signal on MRBTI which was defined as "acute clot sign (ACS)". It has been reported that ACS on MRBTI can be used to predict complete recanalization in CVST patients with endovascular treatment [7].

In a recent multicenter study, endovascular aspiration therapy using large-hole suction catheters for CVST has shown that is a safe and feasible approach to the treatment of anticoagulation refractory CVST [8].

It cannot be treated medically in the case of CVST, decompressive surgery is brought to the agenda, as in other disease states causing intracranial pressure increase, mass effect and herniation. Venous outflow obstruction rapidly causes edema and space-occupying venous infarction, and decompressive craniectomy (DC) can effectively reduce intracranial pressure [9].

CVST may present with a wide range of clinical findings. CVST is a rare but potentially life-threatening disease. Early recognition and appropriate treatment can provide positive results. Outcome is often good and most patients make a full recovery, although a small proportion suffers death or disability [10].

\section{Bibliography}

1. Ferro JM and Aguiar de Sousa D. "Cerebral Venous Thrombosis: an Update". Current Neurology and Neuroscience Reports 19.10 (2019): 74.

2. Kalita J., et al. "Cerebral Venous Sinus Thrombosis Score and its Correlation with Clinical and MRI Findings". Journal of Stroke and Cerebrovascular Diseases 15 (2019): 104324.

3. Mehta A., et al. "Cerebral Venous Thrombosis Headache". Current Pain and Headache Reports 23 (2019): 47.

4. Zhu X., et al. "Transcranial Color-Coded Sonography for the Detection of Cerebral Veins and Sinuses and Diagnosis of Cerebral Venous Sinus Thrombosis". Ultrasound in Medicine and Biology (2019).
5. Aguiar de Sousa D., et al. "Brush Sign Is Associated with Increased Severity in Cerebral Venous Thrombosis". Stroke 50 (2019): 1574-1577.

6. Wasay M., et al. "Asian Study of Cerebral Venous Thrombosis". Journal of Stroke and Cerebrovascular Diseases 24 (2019): 104247.

7. Yang X., et al. "Predictors of successful endovascular treatment in severe cerebral venous sinus thrombosis". Annals of Clinical and Translational Neurology 6 (2019): 755-761.

8. Dandapat S., et al. "Safety and efficacy of the use of large bore intermediate suction catheters alone or in combination for the treatment of acute cerebral venous sinus thrombosis: A multicenter experience". Interventional Neuroradiology (2019).

9. Avanali R., et al. "Role of Decompressive Craniectomy in the Management of Cerebral Venous Sinus Thrombosis". Frontier Neurology 10 (2019): 511.

10. Behrouzi R and Punter M. "Diagnosis and management of cerebral venous thrombosis". Clinical Medicine (Lond) 18 (2018): 75-79.

Volume 2 Issue 10 October 2019

(C) All rights are reserved by Abdurrahman Sönmezler. 